\title{
Effect of relay-priority mechanism on multi-hop wireless sensor networks
}

\author{
Davut Arı a, Musa Çıbuka*, Fikri Ağgün ${ }^{b}$ \\ a Bitlis Eren University, Department of Computer Engineering, TR-13000, Bitlis Turkey \\ b Bitlis Eren University, Vocational School of Adilcevaz, TR-13000, Bitlis Turkey
}

\section{ARTICLE INFO}

Article history:

Received 24 November 2017

Received in revised form 17 December 2017

Accepted 20 December 2017

\section{Keywords:}

Wireless Sensor Networks

Relay-Priority Mechanism

MAC Protocol

End-to-End Delay

\begin{abstract}
A B S T RACT
In multi-hop Wireless Sensor Networks (WSN), sensor nodes which cannot communicate directly with the Coordinator Node $(\mathrm{CN})$ can communicate with $\mathrm{CN}$ thanks to the other joined sensor nodes. The multi-hop WSN structure is preferred for large-scale WSNs and that consist of multiple sensor and CN. As in the networks sensor node count increase, hop count increase as well. Because of this, end-to-end delay increases. Unless it is taken prevention, end-to-end delays reach a level that negatively effects on network performance in multi-hop WSN.

In this study, for multi-hop WSNs, it is aimed to design a new a relay-priority mechanism which will reduce the end-to-end delay. This is a method that will reach the CN with a minimum hop count while joining the node. Thanks to the minimum hop, end-to-end delay is reduced. Performance analysis of this study was done in Riverbed (OPNET) Modeler simulation environment.
\end{abstract}

(C) 2017. Turkish Journal Park Academic. All rights reserved.

\section{Introduction}

Multi-hop Wireless Sensor Networks (WSNs) consist of nodes, which are connected with Coordinator Node (CN) through multiple wireless links (Li et al., 2003). In such networks, sensor nodes (SNs) conduct relay mechanism function as well as their own functions (sensing) performing. With this function, the sensor nodes perform the role of the router while performing their own sensing tasks and transmit the data from the neighbor nodes to the CN. Thanks to this connecting form of SN, nodes which are far from the $\mathrm{CN}$ (outside of the coverage of $\mathrm{CN}$ ) can connect to the CN. Thus, the coverage of the WSN becomes wider (Murdiyat et al., 2014; Murdiyat et al., 2016). It also makes possible to transmit data to farther distances with less energy. Multi-hop WSNs have advantages such as coverage, high data transmission rate, low cost (Jain et al., 2005). In the wide areas, data is collected by consuming less energy. Thanks to this, it is extended the network lifetime (Duan et al., 2011).

Many applications of WSNs are available, such as disaster tracking, security, environmental monitoring and traffic control. In these WSN applications, it is used multi-sensor nodes. For example, hundreds or thousands of sensor nodes are deployed in a large monitoring region for environmental monitoring. It is obvious that increasing the number of sensor nodes in the network makes more complicated the network. Due to the advantages mentioned above, the multi-hop network structure is preferred as a connection form for large-scale WSN applications (Kiri et al., 2006). An example of a multi-hop WSN structure is shown in Figure 1.

\footnotetext{
* Corresponding author.

Tel.: +0 434222 0000; fax: +0 4342229145

E-mail address: mcibuk@gmail.com
} 


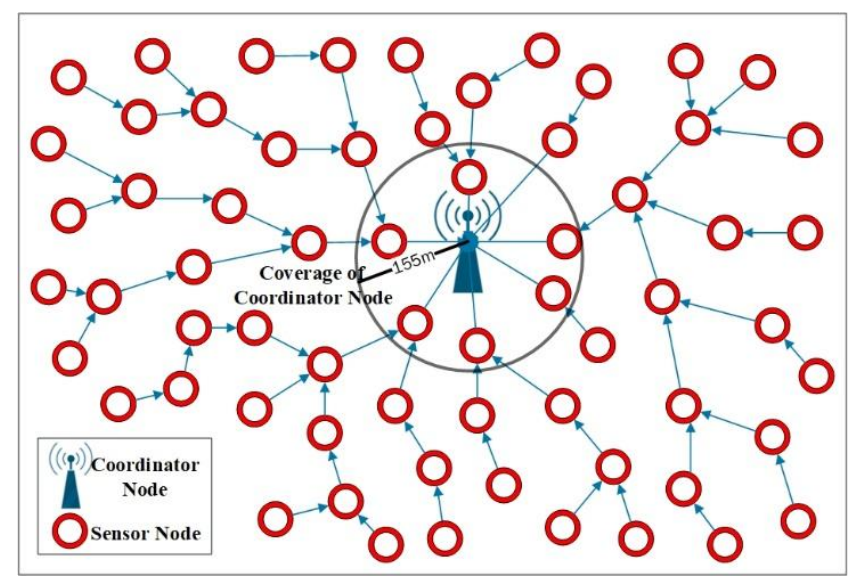

Figure 1. An example of multi-hop Wireless Sensor Network

On the contrary of the above mention advantages of the multi-hop WSN, there are some difficulties (Murdiyat et al., 2016; Jain et al., 2005; Chughtai et al., 2016). End-to-end delay is one of the challenges to be foreseen in the design of multi-hop WSNs. It is obvious that increase in end-to-end delay because of increase in the count of hop in the WSN. The end-to-end delay has a negative impact on the performance of the network. When looking at the literature (Nguyen et al., 2011; Zhao et al., 2008; Nguyen et al., 2010; Lee et al., 2014; Furuta et al., 2010; Xu et al., 2017) the existence of studies can be observed on which contains the solution of end-to-end delay problem. In these studies, endto-end delay solutions were proposed in a built multi-hop WSN network using different methods.

In this study, it is aimed to design a relay-priority mechanism that supplies being connected with least hop to CN while the nodes are joining the network in multi-hop WSNs. Firstly, the node that wants to join the network searches around for a live $\mathrm{CN}$. If a live $\mathrm{CN}$ finds, it sends a request to join directly the network. If it cannot connect directly to the $\mathrm{CN}$, the node sends a relay request to the neighbor nodes that connected to $\mathrm{CN}$. While neighbor nodes answer to this request, they also report that they have reached with how many hops to their CNs. The node accepts the answer of the node that has least hop count among nodes answers. Thus, it is envisaged that the sensor node has a mechanism, which supplies to reaches to $\mathrm{CN}$ with a minimum hop count. With this mechanism, it is aimed to minimize end-to-end delays. The performance of this relay-priority mechanism has been tested in the Riverbed (OPNET) Modeler simulation environment.

As shown in Figure 2, when the nodes wake up, firstly, they broadcast control packets (to search for live $\mathrm{CN}$ ). If there is a $\mathrm{CN}$ in the coverage area of the node, the node joins directly the network. If there is no other user of the channel, the node uses alone as FDMA-based the channel that assigned by the CN. Otherwise, the sensor node uses the channel as time-division (TDMA-based). If the node doesn't find a $\mathrm{CN}$ in the coverage area of itself, it sends a relay request to the neighbor nodes that have connected to $\mathrm{CN}$ to join indirectly the network.

\section{Proposed Relay-Priority Mechanism for Multi- hop Wireless Sensor Networks}

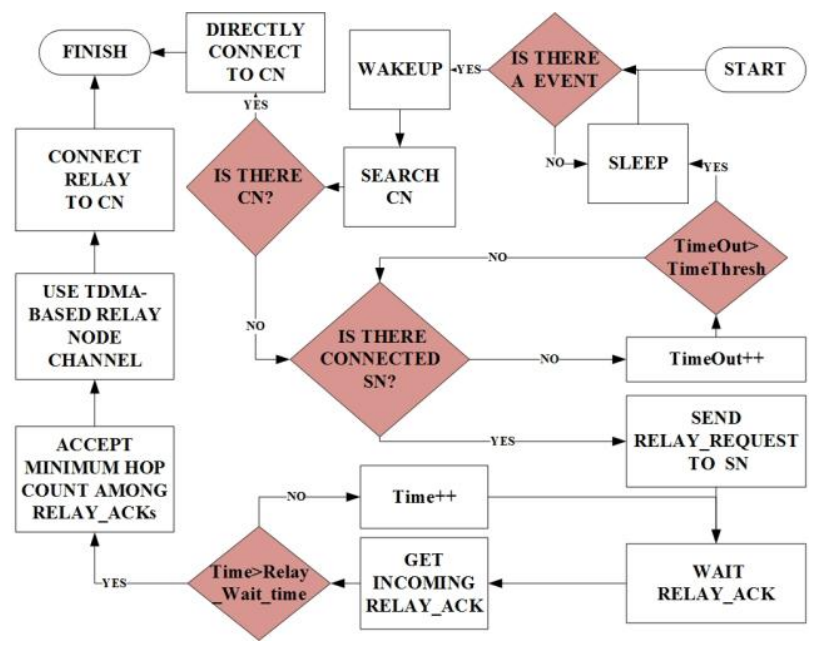

Figure 2. Proposed Relay-Priority Mechanism flowchart

Neighbor nodes that receive this request send Relay_ $A C K$ packet to the requesting node. This package also has information that the node connected to $\mathrm{CN}$ via how many hops counts. The node starts a time counter in order to receive packets from all neighbor nodes at this stage. Until the time counter reaches the threshold value, the requesting node collects the hop count from incoming relay packets.

Start

Initial_Values_Set, Time $=0$, Relay_Wait_time , RELAY_RQUEST, RELAY_ACK;

Step:1 Listen to CN channels

Step:2 If (Is live CN?)

Else GoTo Step5;

Sent RELAY_RQUEST to neighbor SNS GoTo Step3;

Step:3 While(Wait RELAY_ACK packet from neighbor SNs)\{ If(Time $>=$ Relay_ Wait_time) Accept as relay the SN that has

min_hop_count

Send RELAY_ACK to SN GoTo Step4;

Else

Time++; GoTo Step3; \}

Step:4 Use as TDMA-based the channel of relay SN GoTo Step5;

Step:5 I'm joining the network Finish; 
If time counter reaches the threshold value (Relay_Wait_time), the node accepts a relay_ack packet from the node that has minimum hop count.

Then, the requesting node sends a Relay_ACK packet that notifies to accept as a relay node. When the joined node receives the Relay_ACK which packet from requesting node, it sends Relay_Request packet to itself $\mathrm{CN}$ on behalf of the requesting node. If $\mathrm{CN}$ accepts the request, it set channels of the relay node to the requesting node. The requesting node sets to use channels of the relay node. No longer, the requesting nodes communicate with the $\mathrm{CN}$ by using relay node channels TDMA-based method. In this way, it is aimed at the nodes being connected to any $\mathrm{CN}$ with the least hop distance. Thanks to the least hop distance, it is expected that end-to-end delay is minimized. The pseudo-code of this relay-priority mechanism is as shown below.

\section{Simulation and Test}

In this study, Riverbed (OPNET) Modeler simulation environment was preferred because it has had many advantages such as an advanced graphical interface, hierarchical modeling and simultaneously simulations with multiple inputs to test the algorithm. It also has support from very small networks to very large networks (Chang. 1999).

In this study, the node counts were specified as 25,50 and 100 in order to test the end-to-end delay. The coverage area of both CNs and SNs was set to $155 \mathrm{~m}$. Thus, a multi-hop structure was achieved. The simulation time was set to 300th second. In order to track the behavior of special node, it was set the awakening time at 100th second. There are one $\mathrm{CN}$ and a scheduling channel in the network. Network environments have been shown in Figure 3.

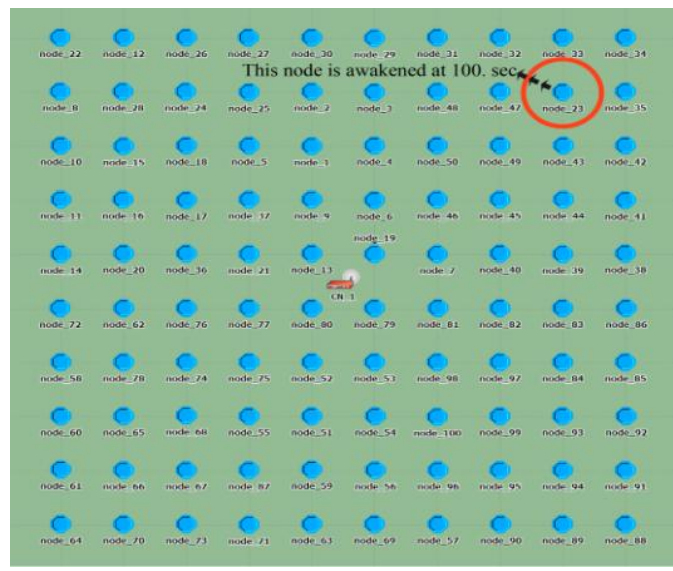

Figure 3 Scenario of 100 nodes in Riverbed Modeler network project environment $\left(1000 \times 1000 \mathrm{~m}^{2}\right)$

\section{Results of Simulation}

In the simulation, firstly, for both the general network and the special a node, the relay-priority mechanism was tested in a 25-node network environment 25 nodes were deployed with $100 \mathrm{~m}$ intervals. The special node is the node awakened at $100^{\text {th }}$ seconds. Although the alternative connection route was few, the mechanism performed well in the 25-node network. It has been given total packet count in Figure 4 and the maximum delay in Figure 5.

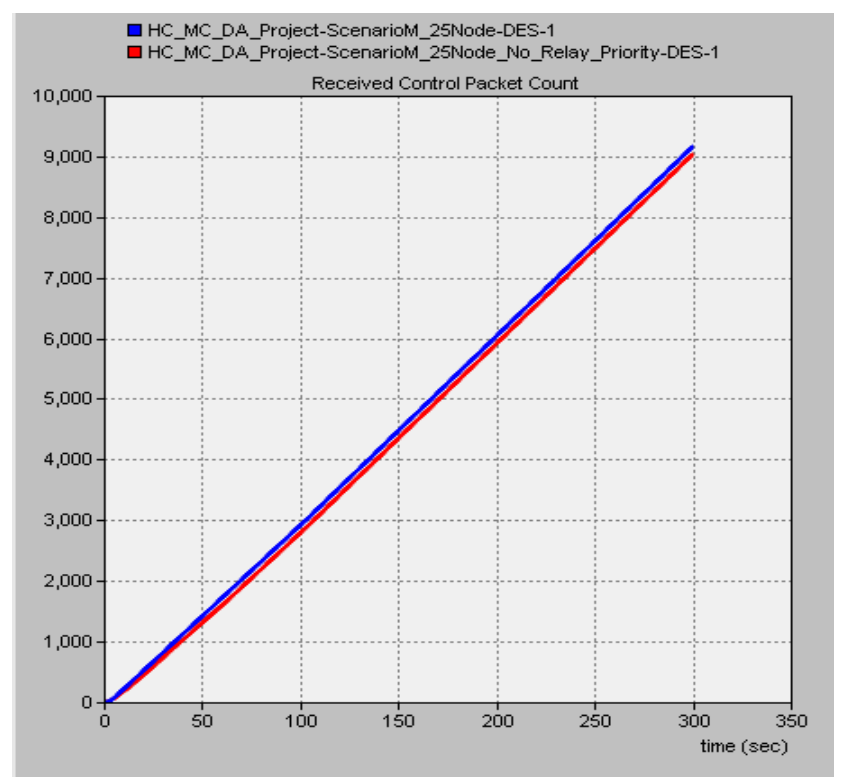

Figure 4. Total number of packets in the 25-nodes network

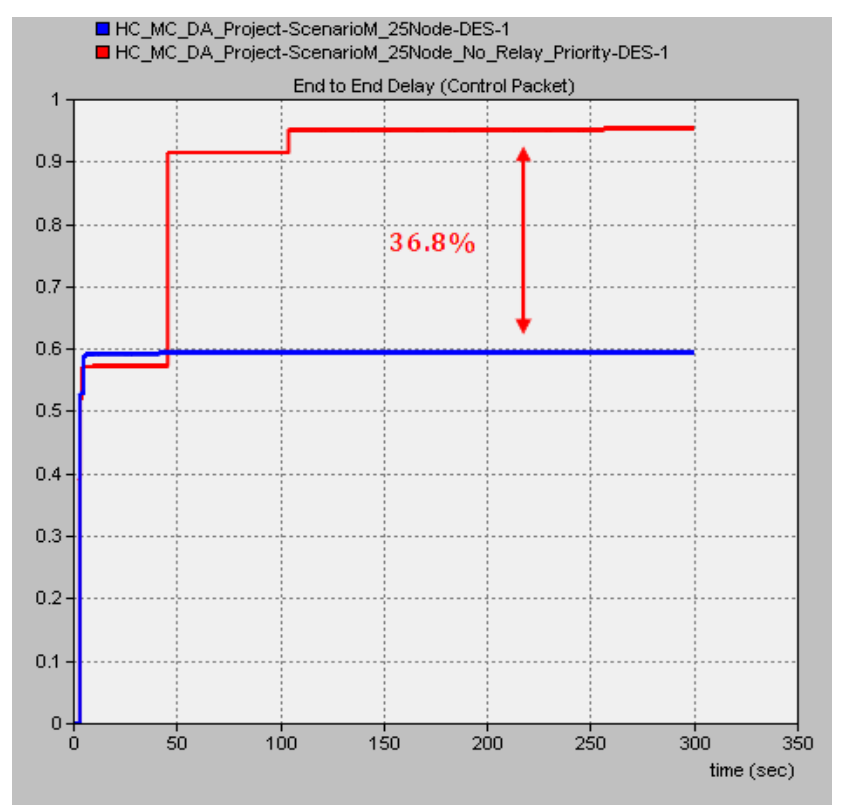

Figure 5. The maximum end-to-end delay in the 25-node network 
In Figure 5, the maximum end-to-end delay in the 25-nodes network was compared according to approximately 9000 packets (see Figure 4). It has been seen that the proposed mechanism reduced by approximately $36.8 \%$ the maximum end-to-end delay. Both in the scenario with priority relaypriority mechanism and in the scenario with non-relaypriority mechanism produced equal package count. It has been given total end-to-end delay Figure 6.

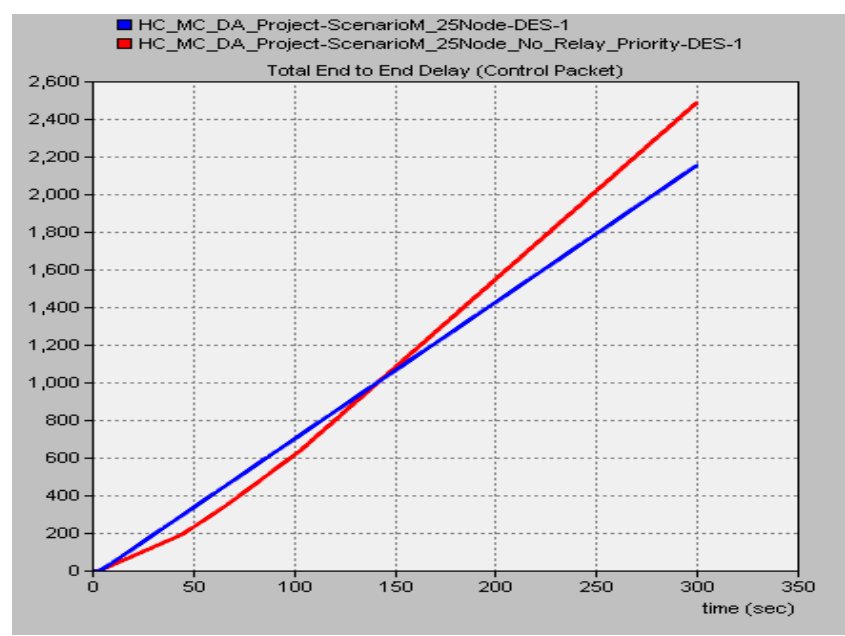

Figure 6. Total end-to-end delay in the 25-node network.

In Figure 6, the total end-to-end delay in the 25-node network was compared according to approximately 9000 packets (see Figure 4). As the time progresses, it has been seen that the difference between graphs has increased. Both in the scenario with priority relay-priority mechanism and in the scenario with non-relay-priority mechanism produced equal package count.

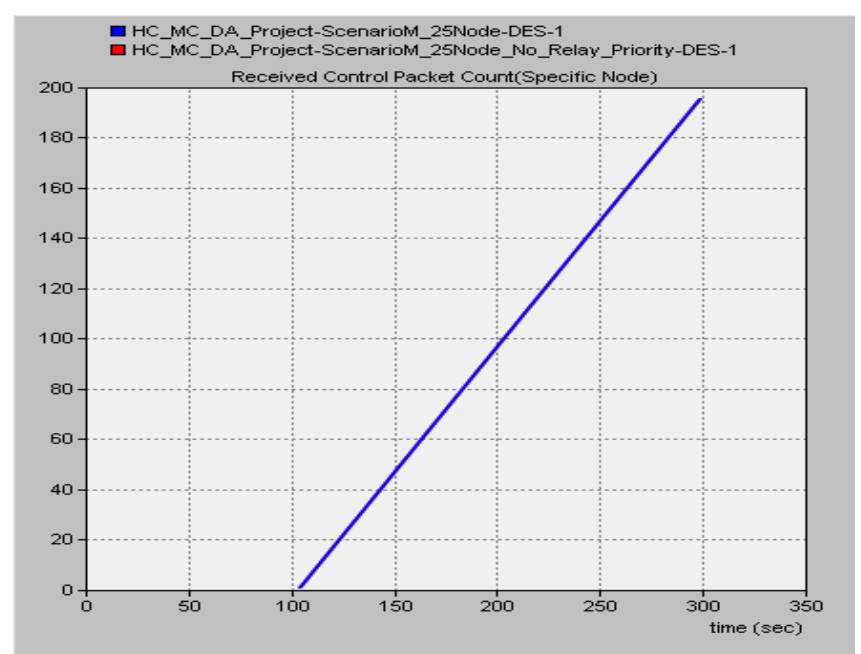

Figure 7. A total number of packets for a specific node.
It has been given the total number of packets for specific node Figure 7 and maximum end-to-end delay for specific node Figure 8.

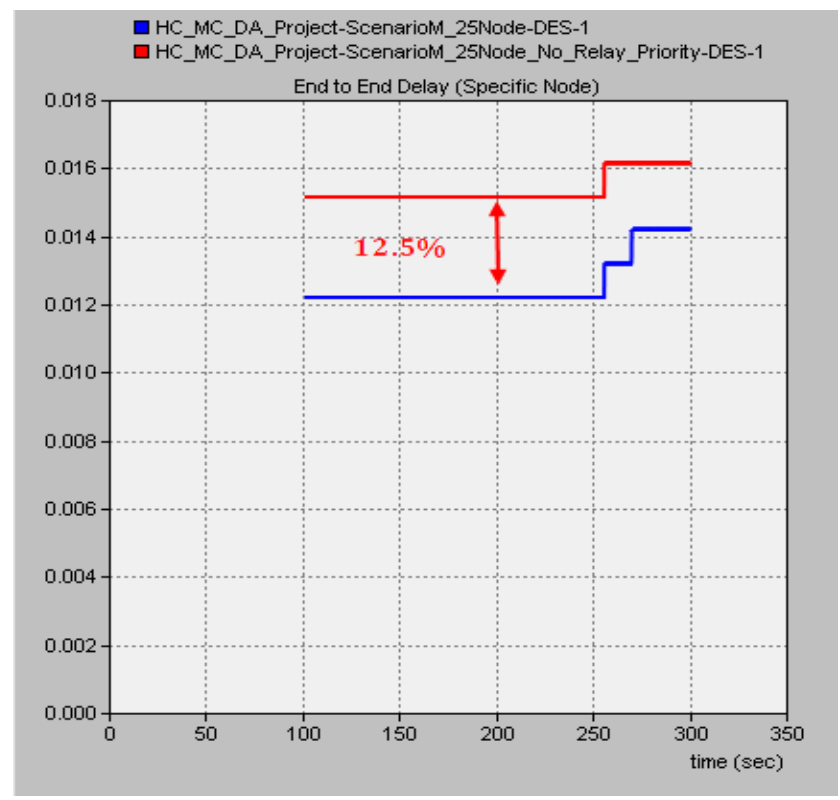

Figure 8. Maximum end-to-end delay for a specific node in the 25-node network.

In Figure 8, the maximum end-to-end delay for the specific node (this node is awakened at $100 \mathrm{sec}$.) in the 25 -node network was compared according to approximately 200 packets (see Figure 7). It has been seen that the maximum end-to-end delay was reduced by approximately $12.5 \%$. Both in the scenario with priority relay-priority mechanism and in the scenario with non-relay-priority mechanism produced equal package count.

It has been given total end-to-end delay for specific node Figure 9. In Figure 9, the total end-to-end delay for a specific node in the 25-node network was compared according to approximately 200 packets (see Figure 7). As the time progresses, it has been seen that the difference between graphs has increased. Both in the scenario with priority relay-priority mechanism and in the scenario with non-relay-priority mechanism produced equal package count.

It has been given Hop count the 25-node network Figure 10. In Figure 10, hop count of relay-priority mechanism and hop count of the non-relay-priority mechanism have been shown for the 25-node network. The maximum hop count has been seen 1 in the scenario of the relay-priority mechanism and 2 in the other scenario. It has been seen that the nodes were selected the least hop. 


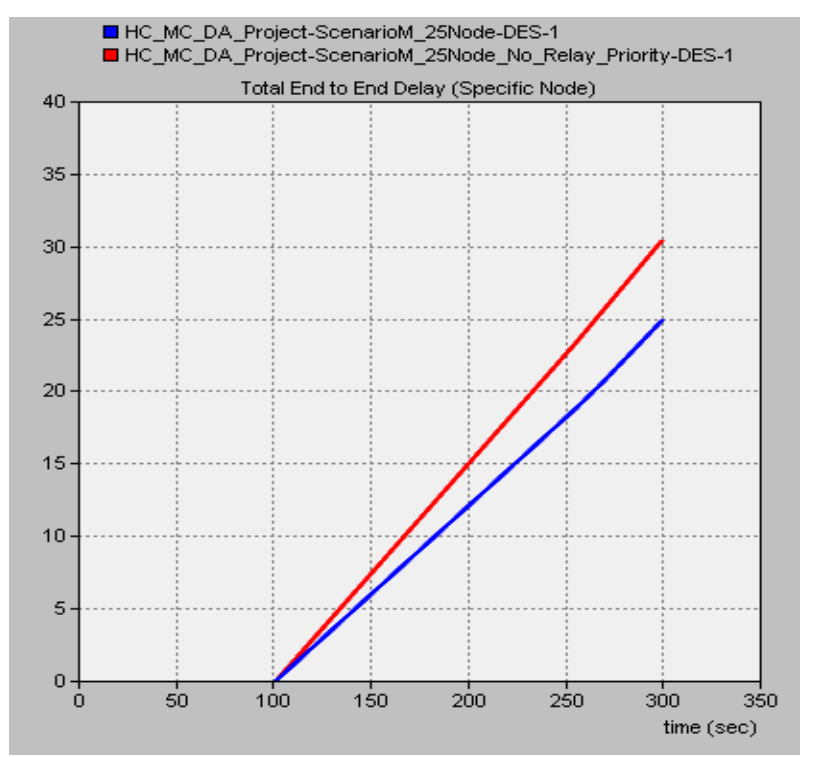

Figure 9. Total end-to-end delay for a specific node.

\begin{tabular}{|c|c|c|}
\hline $\begin{array}{c}\text { parent } \\
\text { name }\end{array}$ & eve & $\begin{array}{c}\text { orijin } \\
\text { name }\end{array}$ \\
\hline & & \\
\hline CN_1 & 0 & CN_1 \\
\hline I CN_1 & 0 & CN_1 \\
\hline | CN-1 & 0 & $\mathrm{CN}^{-} 1$ \\
\hline | CN-1 & 0 & $\mathrm{CN}^{-} 1$ \\
\hline | CN-1 & 0 & $\mathrm{CN}_{-}^{-1}$ \\
\hline ( $\mathrm{CN}^{-} 1$ & 0 & $\mathrm{CN}^{-} 1$ \\
\hline I CN_1 & 0 & CN_1 \\
\hline | CN-1 & 0 & $\mathrm{CN}^{-} 1$ \\
\hline $\mathrm{CN}^{-} 1$ & 0 & $\mathrm{CN}^{-} 1$ \\
\hline | node_2 & 1 & node_2 \\
\hline I node 2 & 1 & node ${ }^{-} 2$ \\
\hline | node_3 & 1 & node_3 \\
\hline | node 32 & 1 & node 32 \\
\hline I node_32 & 1 & node_32 \\
\hline I node_32 & 1 & node 32 \\
\hline | node 1 & 1 & node ${ }^{-} 1$ \\
\hline I node_1 & 1 & node_1 \\
\hline I node 9 & 1 & node 9 \\
\hline I node 9 & 1 & node ${ }^{-} 9$ \\
\hline I node-9 & 1 & node 9 \\
\hline I node_9 & 1 & node_9 \\
\hline I node_50 & 1 & node_50 \\
\hline I node 46 & 1 & node 46 \\
\hline | node 46 & 1 & node 46 \\
\hline node_3 & 1 & node_3 \\
\hline
\end{tabular}

a) Hop count with relaypriority mechanism

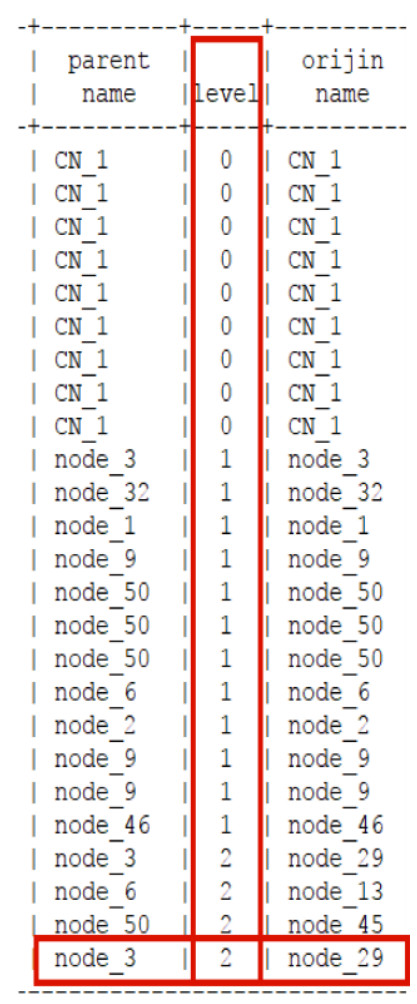

b) Hop count with nonrelay-priority mechanism
Figure 10. Hop count for specific node (node_48) with relaypriority mechanism in the 25 -node network

Secondly, the relay-priority mechanism was tested in a 50node network environment. 50 nodes were deployed with $100 \mathrm{~m}$ intervals. The special node is the node awakened at 100th second. The results of simulation were shown that the relay-priority mechanism works well in 50 nodes WSN.

It has been given the total number of packets for the 50node network in Figure 11 and the maximum end-to-end delay for 50-node network in Figure 12.

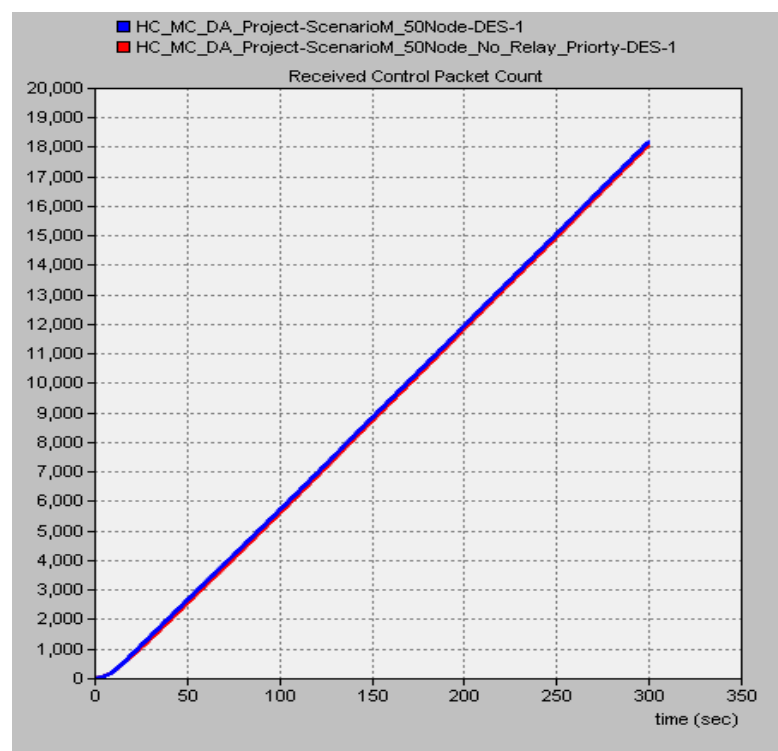

Figure 11. Total number of packets in the 50 -node network

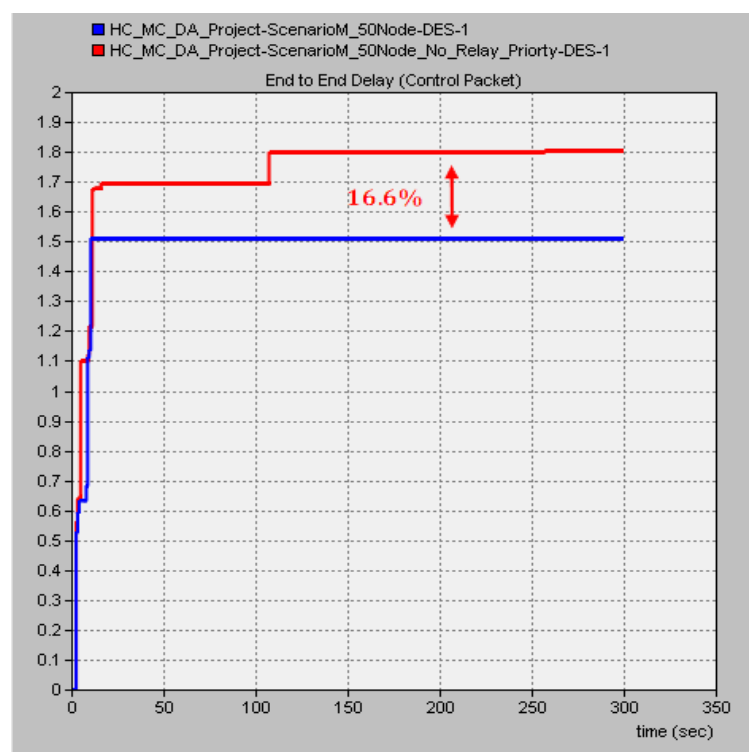

Figure 12. The maximum end-to-end delay in the 50-node network

In Figure 12, the maximum end-to-end delay in the 50-node network was compared according to approximately 18000 packets (see Figure 11). It has been seen that the proposed mechanism reduced by approximately $16.6 \%$ maximum end-to-end delay. Both in the scenario with priority relay- 
priority mechanism and in the scenario with non-relaypriority mechanism produced equal package count. It has been given total end-to-end delay in the 50-node network in Figure 13.

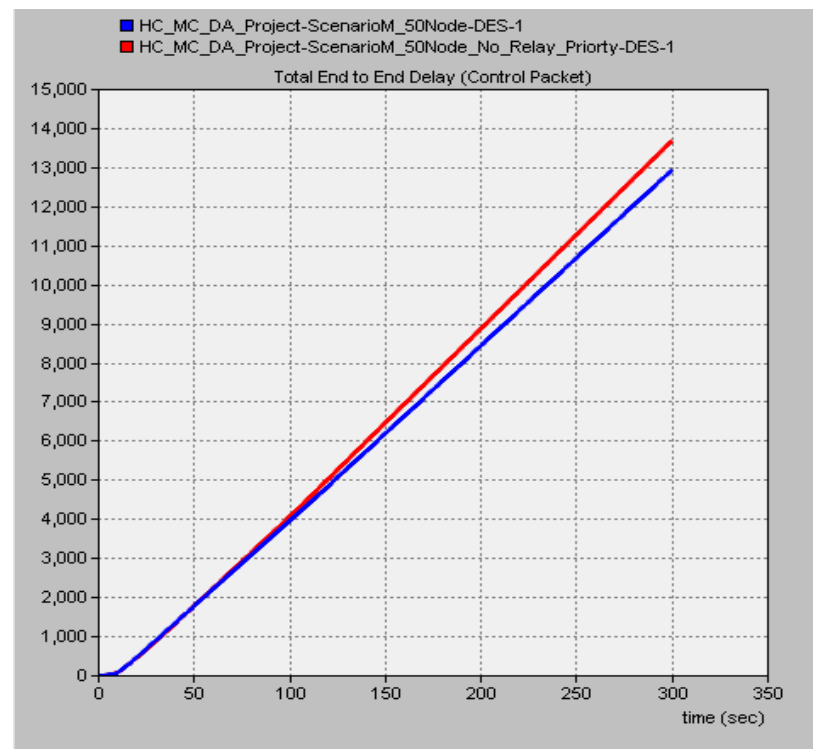

Figure 13. Total end-to-end delay in the 50-node network.

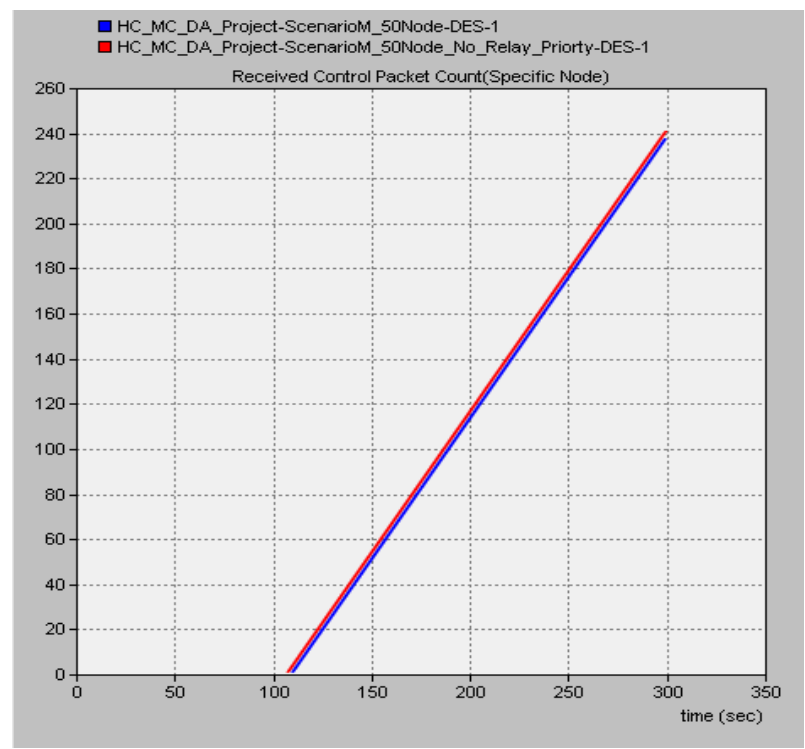

Figure 14. The total number of packets in the 50 -node network

In Figure 13, the total end-to-end delay in the 50-node network was compared according to approximately 18000 packets (see Figure 11). As the time progresses, it has been seen that the difference between graphs has increased. In addition, both in the scenario with priority relay-priority mechanism and in the scenario with non-relay-priority mechanism produced equal package count.
It has been given the total number of packets for a specific node in 50-node network in Figure 14 and maximum endto-end delay for a specific node in 50-node network in Figure 15. In Figure 15, the maximum end-to-end delay for the specific node (this node is awakened at $100 \mathrm{sec}$.) in the 50-node network was compared according to approximately 240 packets (see Figure 14). It has been seen that the maximum end-to-end delay was reduced by approximately $52.1 \%$. Both in the scenario with priority relay-priority mechanism and in the scenario with nonrelay-priority mechanism produced equal package count.

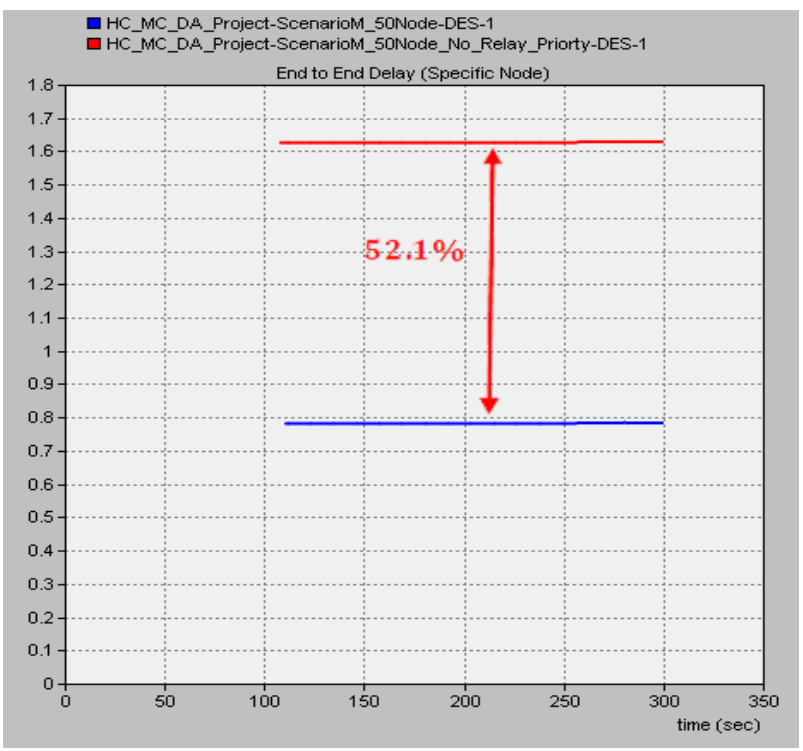

Figure 15. Maximum end-to-end delay for specific node in the 50node network

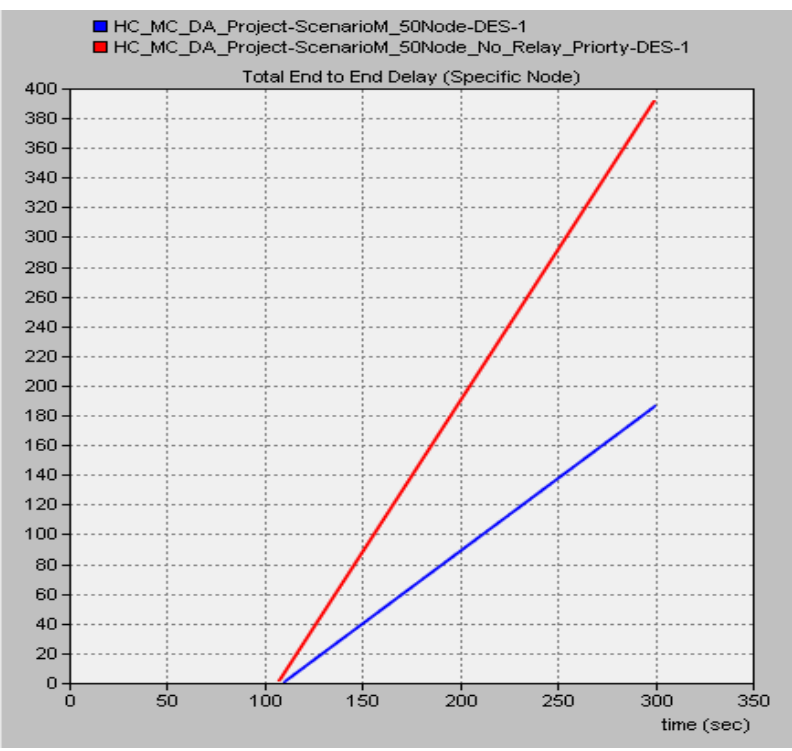

Figure 16. Total end-to-end delay for specific node in the 50-node network 
It has been given total end-to-end delay for specific node in 50 -node network in Figure 16. In Figure 16, the total endto-end delay for a specific node in the 50 -node network was compared according to approximately 240 packets (see Figure 14). In addition, both in the scenario with priority relay-priority mechanism and in the scenario with nonrelay-priority mechanism produced equal package count. It has been given hop count the 50-node network Figure 17.

\begin{tabular}{|c|c|c|}
\hline $\begin{array}{c}\text { parent } \\
\text { name }\end{array}$ & I level & $\begin{array}{c}\text { orijin } \\
\text { name }\end{array}$ \\
\hline CN_1 & 0 & I CN_1 \\
\hline $\mathrm{CN}^{-} \mathrm{-}$ & 0 & I $\mathrm{CN}^{-} 1$ \\
\hline $\mathrm{CN}_{-}^{-1}$ & 0 & I CN_1 \\
\hline node 48 & 1 & I node_48 \\
\hline node_13 & 1 & I node_13 \\
\hline node_13 & 1 & I node_13 \\
\hline node_21 & 1 & I node_21 \\
\hline node_21 & 1 & | node 21 \\
\hline node_1 & 1 & I node_1 \\
\hline node_1 & 1 & I node_1 \\
\hline node_1 & 1 & I node_1 \\
\hline node_18 & 1 & I node_18 \\
\hline node_18 & 1 & I node_18 \\
\hline node_18 & 1 & I node_18 \\
\hline node_17 & 1 & | node_17 \\
\hline node_48 & 1 & I node_48 \\
\hline node_ 48 & 1 & I node_48 \\
\hline node_48 & 1 & I node_48 \\
\hline node_9 & 1 & | node_9 \\
\hline node_13 & 2 & I node_33 \\
\hline node_21 & 2 & I node_42 \\
\hline node_1 & 2 & I node_3 \\
\hline node_18 & 2 & I node_25 \\
\hline node_18 & 2 & | node_24 \\
\hline node_ 48 & 2 & I node_35 \\
\hline node_48 & 2 & I node_35 \\
\hline node_48 & 2 & I node_35 \\
\hline node_9 & 2 & I node_4 \\
\hline node_13 & 2 & I node_19 \\
\hline node_13 & 2 & I node_33 \\
\hline node_21 & 2 & I node_38 \\
\hline node_21 & 2 & I node_42 \\
\hline node_1 & 2 & I node_6 \\
\hline node_1 & 2 & I node_3 \\
\hline node_1 & 2 & I node_3 \\
\hline node_1 & 2 & I node_3 \\
\hline node_18 & 2 & I node_28 \\
\hline node_18 & 2 & I node_28 \\
\hline node_18 & 2 & I node_28 \\
\hline node_18 & 2 & | node_28 \\
\hline node_17 & 2 & I node_15 \\
\hline node_48 & 2 & | node_16 \\
\hline node_48 & 2 & I node_16 \\
\hline node 48 & 2 & I node 16 \\
\hline node_1 & 2 & I node_3 \\
\hline
\end{tabular}

a) Hop count (level) with relay-priority mechanism

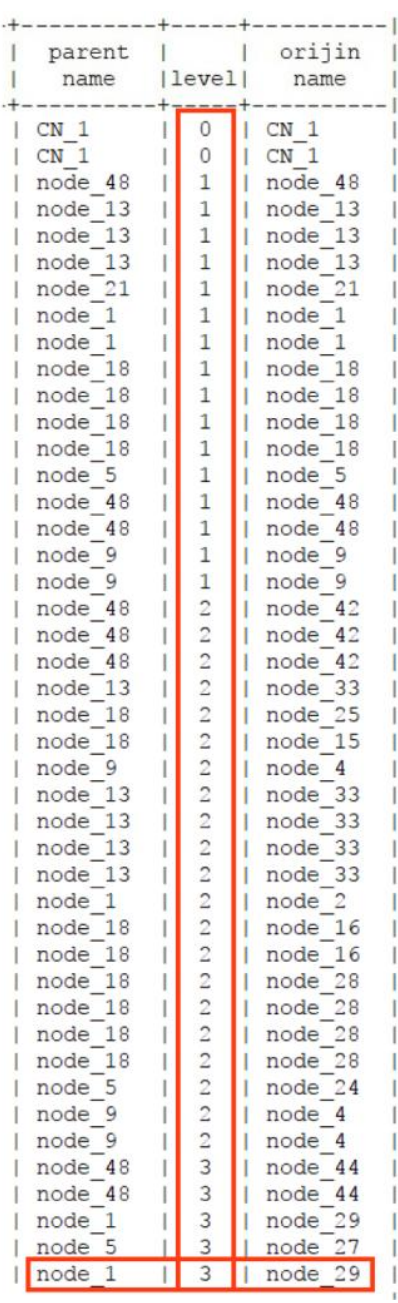

b) Hop count (level) with nonrelay-priority mechanism
Figure 17. Hop count (level) in the 50-node network

In Figure 17, hop count of relay-priority mechanism and hop count of the non-relay-priority mechanism have been shown for the 50-node network. The maximum hop count has been seen 2 in the scenario of the relay-priority mechanism and 3 in the other scenario. It has been seen that the nodes were selected the least hop.

Thirdly, the relay-priority mechanism was tested in a 100node network environment. As shown in Figure 3, 100 nodes were deployed with $100 \mathrm{~m}$ intervals. node_23 was selected as a special node. The special node is the node awakened at 100th seconds. The results of simulation have shown that the relay-priority mechanism worked well in 100 nodes WSN. It has been given a total number of packets for 100-node network in Figure 18 and Maximum end-toend delay for 100-node network in Figure 19.

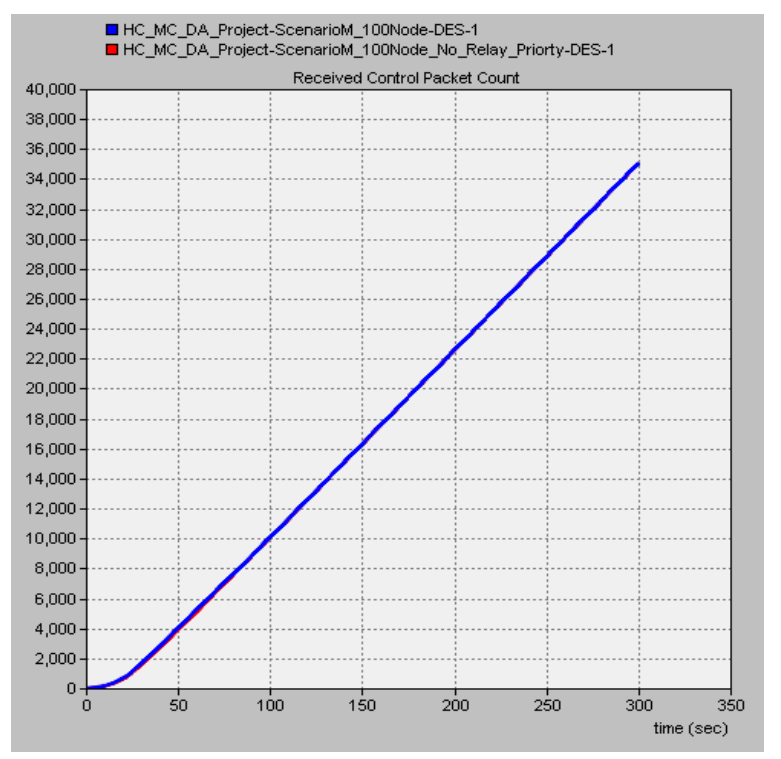

Figure 18. The total number of packets in the 100-node network

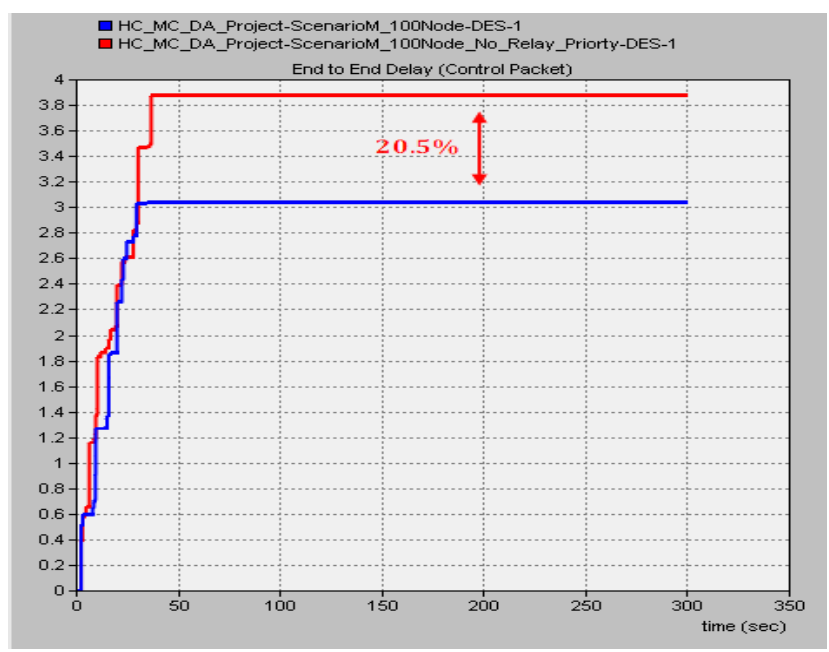

Figure 19. The maximum end-to-end delay in the 100-node network

In Figure 19, the maximum end-to-end delay in the 100node network was compared according to approximately 35000 packets (see Figure 18). It has been seen that the proposed mechanism reduced approximately 20.5\% maximum end-to-end delay. 
It has been given total end-to-end delay in 100-node network in Figure 20.

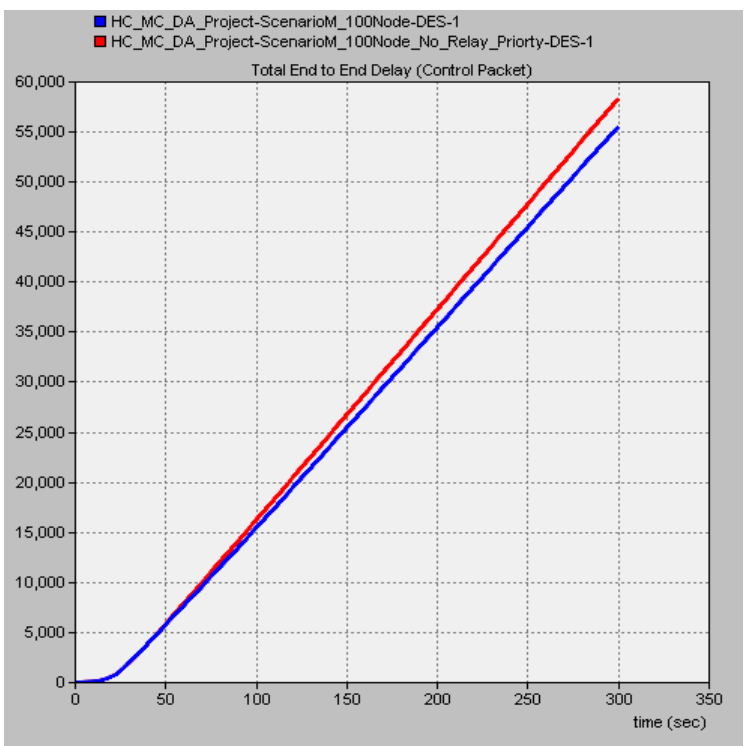

Figure 20. Total end-to-end delay in the 100-node network

In Figure 20, the total end-to-end delay in the 100-node network was compared according to approximately 35000 packets (see Figure 18). It has been seen that the difference between the two graphs increased. This result has shown that the end-to-end delay reduced thanks to the relaypriority mechanism.

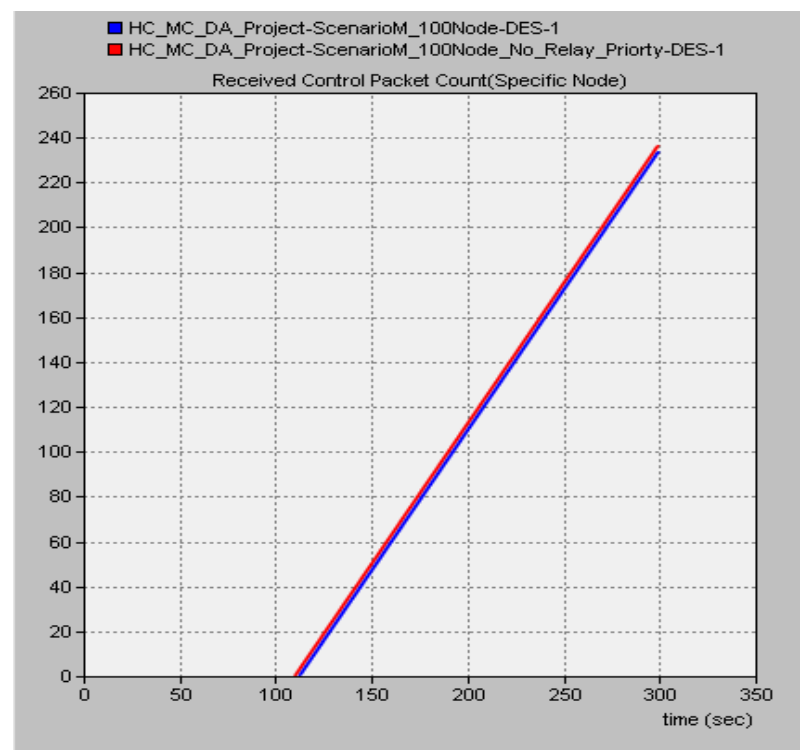

Figure 21. The total number of packets for specific node in the 100-node network

It has been given a total number of packets for a specific node in 100-node network in Figure 21 and maximum end- to-end delay for a specific node in 100-node network in Figure 22.

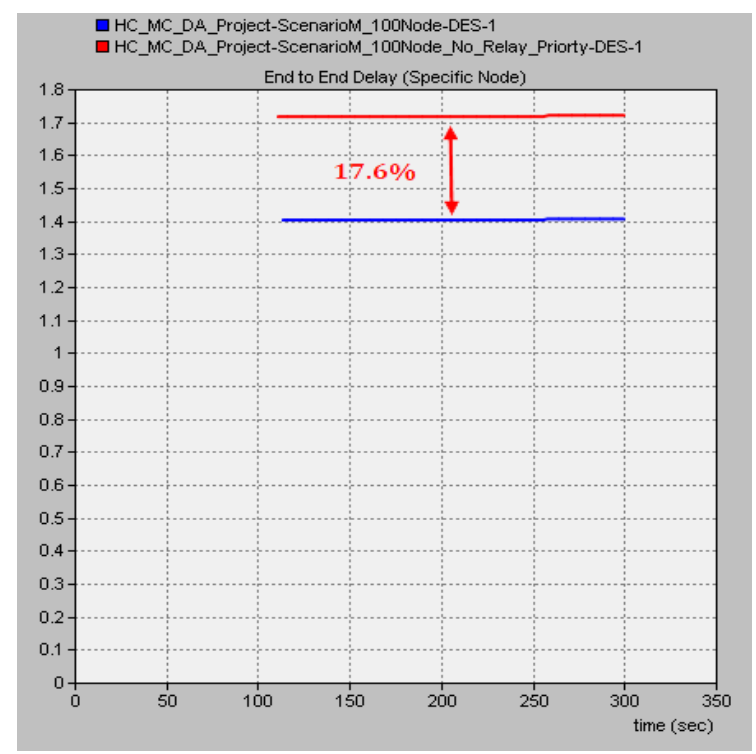

Figure 22. Maximum end-to-end delay for specific node in the 100node network

In Figure 22, the maximum end-to-end delay for the specific node (this node was awakened at $100 \mathrm{sec}$.) in the 100 -node network was compared according to approximately 240 packets (see Figure 21). It has been seen that the maximum end-to-end delay was reduced by approximately $17.6 \%$.

It has been given total end-to-end delay for specific node in 100-node network in Figure 23

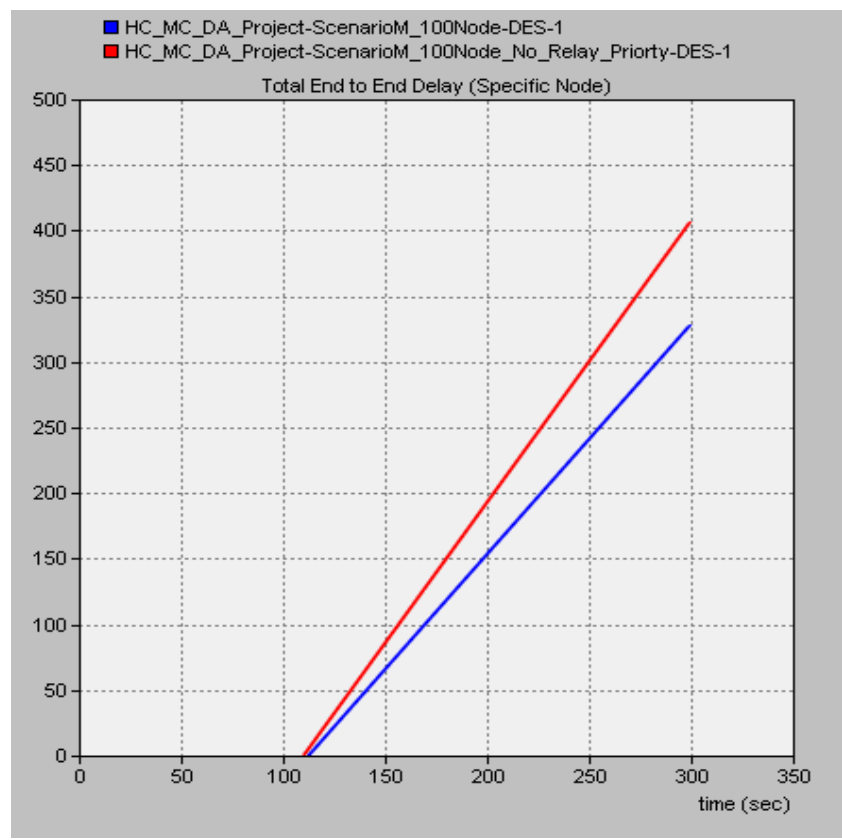

Figure 23. Total end-to-end delay for specific node 
In Figure 23, the total end-to-end delay for a specific node in the 100-node network was compared according to approximately 240 packets (see Figure 21). It has been seen that the difference between the two graphs increased. This result has shown that the total end-to-end delay reduced thanks to the relay-priority mechanism.

\section{Conclusions}

In this work, the relay-priority mechanism was applied to a multi-hop WSNs consisting of static nodes. This mechanism was intended to reduce the delay problem in multi-hop wireless sensor networks. The results have shown that the nodes preferred the shorter hop path. Thus, the end-to-end delay has been reduced. In this study, it has been proposed a solution to the end-to-end delay by using medium access methods (MAC).

In the future studies, it is aimed that this mechanism will be adapted for mobile-WSNs.

\section{References}

Chang, X., 1999. Network simulations with OPNET. In: Simulation Conference Proceedings, 1999 Winter, IEEE, p. 307-314.

Chughtai, O., Badruddin, N., and Awang, A., 2016. A novel congestion alleviation procedure in multi-hop wireless sensor networks. In Intelligent and Advanced Systems (ICIAS), 2016 6th International Conference on, IEEE, p. 1-6.

Duan, C., Shi, F., Ding, X., Xiao, X., and Duan, P., 2011. A novel TDMA and multi-hop MAC protocol in cluster-based wireless sensor networks. In Industrial Electronics and Applications (ICIEA), 2011 6th IEEE Conference on, IEEE, p. 805-808.

Furuta, T., Sasaki, M., Ishizaki, F., Ukai, T., Miyazawa, H., and Koo, W., 2010. New formulation for scheduling problem in multi-hop wireless sensor networks. In Proceedings of the 6th International Wireless Communications and Mobile Computing Conference, ACM, p. 73-78.

Jain, K., Padhye, J., Padmanabhan, V. N., and Qiu, L., 2005. Impact of interference on multi-hop wireless network performance. Wireless networks, 11, 471-487.

Kiri, Y., Sugano, M., and Murata, M., 2006. Performance Evaluation of Intercluster Multi-hop Communication Large-Scale Sensor Networks. In Computer and Information Technology, 2006. CIT'06. The Sixth IEEE International Conference on, IEEE, p. 215-215.

Lee, J. W., and Cho, H. S., 2014. Cascading multi-hop reservation and transmission in underwater acoustic sensor networks. Sensors, 14, 18390-18409.

Li, L. E., and Sinha, P., 2003. Throughput and energy efficiency in topology-controlled multi-hop wireless sensor networks. In Proceedings of the 2nd ACM international conference on Wireless sensor networks and applications, ACM, p.132-140.

Murdiyat, P., Chung, K. S., and Chan, K. S., 2014. Predicting the network throughput of wide area WSN in rural areas. In Communications (APCC), 2014 Asia-Pacific Conference on, IEEE, p. 106-111.

Murdiyat, P., Chung, K. S., and Chan, K. S., 2016. A multi-channel MAC for multi-hop wireless sensor networks minimizing hidden node collision. In Communications (APCC), 2016 22nd Asia-Pacific Conference on, IEEE, p. 535-540.

Nguyen, K., and Ji, Y., 2010. AM-MAC: an energy efficient, Adaptive Multi-hop MAC protocol for sensor networks. In Proceedings of the 6th International Wireless Communications and Mobile Computing Conference, ACM, p. 432-436.

Nguyen, K., and Ji, Y., 2011. Achieving minimum latency in multihop mac protocol for wireless sensor networks. In Vehicular Technology Conference (VTC Spring), 2011 IEEE 73 ${ }^{\text {rd }}$, IEEE, p. 1-5.

Xu, W., Cheng, W., Zhang, Y., Shi, Q., and Wang, X., 2017. On the Optimization Model for Multi-Hop Information Transmission and Energy Transfer in TDMA-Based Wireless Sensor Networks, IEEE Communications Letters, 215, 1095-1098.

Zhao, J. J., and Sun, X., 2008. MAC protocol based on T-MAC multihop reservation for the short-latency wireless sensor network. In Communication Technology, 2008. ICCT 2008. 11th IEEE International Conference on, IEEE, p. 114-117. 